\title{
Trigeminal autonomic cephalalgias: frequency in a general neurology clinic setting
}

\author{
A. J. Larner
}

Received: 13 May 2008/Accepted: 15 June 2008/Published online: 2 July 2008

(C) Springer-Verlag 2008

\begin{abstract}
Cases of trigeminal autonomic cephalalgia seen in general neurology outpatient clinics by one consultant neurologist over a 4 year period (2004-2007 inclusive) were identified. A total of 40 cases was identified $(=1.2 \%$ of all referrals, $5.3 \%$ of headache referrals), most with cluster headache, the remainder with SUNCT or SUNA. There was evidence of both delayed diagnosis and inappropriate treatment, despite involvement of other secondary care specialties. Increased awareness of this diagnostic category is required to ensure effective deployment of effective, evidence-based treatments.
\end{abstract}

Keywords Trigeminal autonomic cephalalgia .

Diagnosis · Frequency · Referral source - SUNCT ·

Cluster headache

\section{Introduction}

One of the changes introduced in the second edition of the International Classification of Headache Disorders (ICHD2) developed by the International Headache Society was the concept of trigeminal autonomic cephalalgias (TACs) [1]. This category of primary headache disorders encompasses cluster headache, paroxysmal hemicrania, shortlived unilateral neuralgiform headache attacks with conjunctival tearing (SUNCT), and probable trigeminal autonomic cephalalgia. These disorders are characterised clinically by unilateral head and face pain which may be of

\footnotetext{
A. J. Larner $(\bowtie)$

Walton Centre for Neurology and Neurosurgery, Lower Lane, Fazakerley, Liverpool L9 7LJ, UK

e-mail: a.larner@thewaltoncentre.nhs.uk
}

excruciating severity, with associated ipsilateral autonomic features [2]. The correct identification of TACs is important because their treatment differs from that of other primary headache disorders, such as migraine and tensiontype headache [2-4].

In the UK, dedicated headache clinics are relatively few and hence many patients with TACs, along with other headache types, are referred to general neurological outpatient clinics. A prospective study to measure both the frequency of TACs in this setting and the origin of referrals was undertaken.

\section{Materials and methods}

Data were collected prospectively on all patients seen by one consultant neurologist in general neurology outpatient clinics at two district general hospitals and a regional neuroscience centre over a 4-year period (2004-2007 inclusive). All cases of headache were classified according to ICHD-2 criteria [1]. Patients with short-lasting unilateral neuralgiform headache attacks with cranial autonomic features (SUNA) were differentiated from SUNCT [5].

\section{Results}

Over the 4 year period, 3395 new patient referrals were seen in total. Of these, headache was the reason for referral and/or the principal patient complaint in $795(=23.4 \%)$. Of these 795,42 were diagnosed with a TAC $(=5.3 \%$ of all headaches, $1.2 \%$ of all referrals; range $=5-11$ cases per annum). These 42 referrals comprised 40 patients $(\mathrm{M}: \mathrm{F}=27: 13)$, two patients being referred on two occasions over the 4-year period. 
Referral source of TAC patients was most often a primary care (general) practitioner $(n=32)$. Of those referred from secondary care, the majority were sent from ophthalmologists (5/8) and comprised a significant proportion, nearly one-fifth, of all ophthalmological referrals to the general neurology clinic during this time period. Referrals were also received from general physicians (2) and an ENT surgeon (1). The total cohort of headache patients included a number $(n=11)$ referred under the auspices of UK Department of Health guidelines for CNS/brain tumour ("2-week" referrals), none of whom received a TAC diagnosis.

The majority of TAC patients were diagnosed with cluster headache $(\mathrm{CH} ; n=36, \quad \mathrm{M}: \mathrm{F}=24: 12$; age range $=21-82$ years, mean $43.8 \pm 13.4$ years $)$. Most cases were episodic $\mathrm{CH}$ (35) rather than chronic $\mathrm{CH}$ (1). Three patients reported diagnosing themselves by means of self-directed searching for medical information on the internet. Three others reported having presented themselves at some time to a hospital emergency department because of their headaches. Two patients reported undergoing dental extractions in the belief that pain was of dental origin prior to the diagnosis of $\mathrm{CH}$ being made.

Four patients had either SUNCT or SUNA; three cases were primary, one was symptomatic, namely a patient in whom SUNCT was induced by taking dopamine agonist medication prescribed for a presumed pituitary microprolactinoma. This patient self-diagnosed by means of selfdirected internet searching for medical information.

No patients were identified with paroxysmal hemicrania. Cases of hemicrania continua were seen $(n=3$; all chronic rather than episodic) but were not included in the analysis since this entity is not currently classified with the TACs [1], although there may be shared pathophysiological features $[2,6]$.

\section{Discussion}

The percentage of all headache patients in this cohort of general neurological outpatient referrals (23.4\%) was similar to that seen in a previous large, non-overlapping, cohort from the same sources $(20.8 \%)$ [7], suggesting that this is representative of clinical practice in this geographical area, and notwithstanding the inception of a dedicated headache clinic at the regional neuroscience centre in the meantime.

TACs were not common amongst general neurology outpatient clinic referrals (5-11 cases per annum), with $\mathrm{CH}$ accounting for the majority of cases. TAC frequency $(5.3 \%$ of all headache patients) was almost identical to the frequency of $\mathrm{CH}$ cases seen in a UK specialist headache clinic (5\%) over a 3-year period (1997-2000) [8], suggesting the possibility that TACs may be as common in general neurology as in specialist headache clinics. TAC frequency (1.2\% of all referrals) was roughly three times that of primary headache associated with sexual activity $(0.42 \%)$ [9], another acute primary headache disorder. TACs may be relatively common amongst patients with headache who attend at emergency departments (11.5\% in one series) [10].

Since treatment recommendations for TACs differ from those for migraine and tension-type headaches [2-4], correct diagnosis is key to initiating appropriate treatment. The data reported here suggest that a high index of clinical suspicion is necessary to identify cases in the general neurology clinic because of their relative infrequency. Cases may also be seen by ophthalmologists, ENT surgeons, oral surgeons, and general and emergency department physicians. Because TACs are one of the most painful conditions known, causing high levels of distress and functional impairment, all these practitioners should, like neurologists, have some awareness of these conditions to ensure appropriate referral, diagnosis and treatment.

Conflict of interest None.

\section{References}

1. International Headache Society Classification Subcommittee (2004) The international classification of headache disorders, second edition. Cephalalgia 24(Suppl 1):1-160

2. Matharu MS, Goadsby PJ (2008) Trigeminal autonomic cephalalgias: diagnosis and management. In: Silberstein SD, Lipton RB, Dodick DW (eds) Wolff's headache and other head pain, 8th edn. Oxford University Press, Oxford, pp 379-430

3. May A, Leone M, Afra J et al (2006) EFNS guidelines on the treatment of cluster headache and other trigeminal-autonomic cephalalgias. Eur J Neurol 13:1066-1077

4. British Association for the Study of Headache (2007) Guidelines for all healthcare professionals in the diagnosis and management of migraine, tension-type, cluster and medication-overuse headache. BASH, London, pp 40-44

5. Cohen AS, Matharu MS, Goadsby PJ (2006) Short-lasting unilateral neuralgiform headache attacks with conjunctival injection and tearing (SUNCT) or cranial autonomic features (SUNA)-a prospective clinical study of SUNCT and SUNA. Brain 129:2746-2760

6. Matharu MS, Cohen AS, McGonigle DJ et al (2004) Posterior hypothalamic and brainstem activation in hemicrania continua. Headache 44:747-761

7. Larner AJ (2003) NHS Direct for headache. J Neurol Neurosurg Psychiatry $74: 1698$

8. Dowson AJ (2003) Analysis of the patients attending a specialist UK headache clinic over a 3-year period. Headache 43:14-18

9. Larner AJ (2006) Late presentation of primary headache associated with sexual activity: is non-invasive angiography worthwhile? J Headache Pain 7:139-140

10. Gahir KK, Larner AJ (2006) Primary headache disorder in the emergency department: perspective from a general neurology outpatient clinic. Emerg Med J 23:135-136 